03

\title{
О спиновом моменте поверхностной волны на границе раздела гиперболического и топологического изоляторов
}

\author{
(С) А.И. Маймистов, Е.И. Ляшко \\ Национальный исследовательский ядерный университет МИФИ, \\ 115409 Москва, Россия \\ e-mail: aimaimistov@gmail.com
}

Поступила в редакцию 11.08.2018 г.

Обычно поверхностная волна, бегущая вдоль раздела изотропных сред, обладает спиновым моментом, лежащим в плоскости раздела и перпендикулярным направлению распространения. Здесь показано, что если одна из сред является топологическим изолятором, вектор спинового момента имеет компоненту, нормальную к поверхности радела и пропорциональную целым нечетным числам. Появление нормальной компоненты спинового момента связано с топологическим магнитоэлектрическим эффектом, в результате которого поляризация волны меняется при переходе через границу раздела.

DOI: $10.21883 /$ OS.2018.12.46940.235-18

\section{Введение}

Среди характеристик электромагнитного поля наряду с потоком энергии, импульсом важной характеристикой является угловой момент поля. Исследования углового момента вызваны интересом к формированию пучков света с фазовыми дефектами волнового фронта излучения и изучению возможности передачи момента вращения от поля к материальным телам. Это стало особенно актуально с появлением и развитием нанотехнологии. В интегральной оптике и плазмонике роль пучков играют поверхностные волны. Естественно, возник интерес к изучению углового момента, переносимого поверхностной волной.

Полный угловой момент электромагнитного поля относительно точки с радиусом-вектором $\mathbf{r}_{0}$ определяется [1-5] выражением

$$
\mathbf{J}\left(\mathbf{r}_{0}\right)=\int\left(\mathbf{r}-\mathbf{r}_{0}\right) \times \mathbf{P} d V
$$

где $\mathbf{P}$ - плотность импульса, определенная формулой $\mathbf{P}=(4 \pi c)^{-1} \mathbf{E} \times \mathbf{H}$. В однородном изотропном пространстве этот вектор может быть разложен на две части: $\mathbf{J}\left(\mathbf{r}_{0}\right)=\mathbf{L}\left(\mathbf{r}_{0}\right)+\mathbf{S}_{\mathrm{in}}$, где

$$
\mathbf{L}\left(\mathbf{r}_{0}\right)=(4 \pi c)^{-1} \sum_{j} \int E_{j}\left[\left(\mathbf{r}-\mathbf{r}_{0}\right) \times \nabla\right] A_{j} d V
$$

— орбитальный угловой момент поля и

$$
\mathbf{S}_{\mathrm{in}}=(4 \pi c)^{-1} \int \mathbf{E} \times \mathbf{A} d V
$$

— внутренний угловой момент поля, который не зависит от выбора точки $\mathbf{r}_{0}$ и характеризует внутренние свойства самого электромагнитного поля. Другое его название спиновый момент поля.
Вектор А - векторный потенциал, который будет здесь выбран в кулоновской калибровке:

$$
\begin{gathered}
\operatorname{div} \mathbf{A}=0 \\
\mathbf{H}=\operatorname{rot} \mathbf{A}, \quad \mathbf{E}=-\frac{1}{c} \frac{\partial}{\partial t} \mathbf{A} .
\end{gathered}
$$

Используя язык квантовой электродинамики, говорят, что спиновый момент складывается из спиральностей фотонов. Если число фотонов, имеющих различные спиральности, различно, то волна будет циркулярно поляризована и такая волна несет спиновый момент. Следовательно, у линейно поляризованной плоской волны нет спинового углового момента. В пространственно неоднородной среде, где фронт волны не плоский, волна обладает орбитальным и спиновым моментами. Но оказывается, что в случае полного внутреннего отражения неоднородная (эванесцентная) волна обретает спиновый момент, хотя может быть плоской волной [6,7]. Поверхностные волны являются как бы склеенными вдоль плоской границы раздела эванесцентными волнами и переносят как орбитальный, так и спиновый моменты [7-10]. Для случая поверхностной ТМ-волны, распространяющейся вдоль поверхности раздела вакуума и отрицательно преломляющей среды, спиновый момент ненулевой. Но в случае поверхностной ТЕ-волны спиновый момент равен нулю. В [8] было показано, что спиновый угловой момент направлен ортогонально волновому вектору поверхностной волны.

Для существования поверхностной волны необходимо, чтобы диэлектрическая проницаемость одной из сред была отрицательной. Это условие выполняется для поверхности металла, и тогда говорят о поверхностном плазмон-поляритоне $[11,12]$. Отрицательной диэлектрической проницаемостью обладают среды с диэлектрической проницаемостью, близкой к нулю [13-16], и гиперболические среды $[17,18]$. Далее будет рассмотрен случай гиперболической среды. Но получить обобщение 
на другие случаи несложно. Принципиальную роль играет топологический изолятор $[19,20]$. При прохождении границы раздела различных топологических изоляторов (и обычных диэлектриков) происходит поворот векторов магнитного и электрического полей [20-23]. По этой причине нет отдельно поверхностных ТЕ- или ТМволн, а поверхностная волна является гибридной все компоненты полей ненулевые. Поперечное распределение полей поверхностных волн и потоки энергии, переносимые ими, были исследованы в [22-26].

В настоящей работе будут рассмотрены граница раздела топологического изолятора и гиперболического диэлектрика и угловой момент поверхностной волны, распространяющейся вдоль этой границы. Вычислен угловой спиновый момент поверхностной волны, который имеет нормальную компоненту, помимо касательной проекции, типичной для поверхностных плазмонполяритонов. Присутствие топологического изолятора проявляется также в дискретности компонент спинового момента. Получить обобщение на другие случаи оптически линейных или нелинейных сред несложно и будет сделано позже.

\section{Роль плоской границы раздела}

Пусть система координат выбрана так, что ось $X$ направлена нормально к поверхности раздела двух сред. Оси $Y$ и $Z$ лежат в плоскости раздела. Ось $Z$ выбрана вдоль вектора распространения поверхностной волн. В такой системе координат напряженности всех полей зависят только от переменной $z$ и времени [27]. В этом случае для компонент электрического поля $E_{y}$ и магнитного поля $H_{y}$ можно записать волновые уравнения, а остальные компоненты выразить через $E_{y}$ и $H_{y}$. Система уравнений Максвелла сводится к системе уравнений для фурье-компонент напряженностей полей:

$$
\begin{gathered}
\frac{\partial^{2} E_{y}}{\partial x^{2}}+\frac{\partial^{2} E_{y}}{\partial z^{2}}+k_{0}^{2} \mu D_{y}=0 \\
H_{z}=-\frac{i}{k_{0} \mu} \frac{\partial E_{y}}{\partial x}, \quad H_{x}=\frac{i}{k_{0} \mu} \frac{\partial E_{y}}{\partial z}, \\
\frac{\partial E_{x}}{\partial z}-\frac{\partial E_{z}}{\partial x}=i k_{0} \mu H_{y}, \quad D_{z}=\frac{i}{k_{0}} \frac{\partial H_{y}}{\partial x}, \quad D_{x}=-\frac{i}{k_{0}} \frac{\partial H_{y}}{\partial z},
\end{gathered}
$$

где $k_{0}=\omega / c$. Поскольку в направлении $z$ среды однородные, все напряженности полей и индукция представляются в следующем виде: $\mathbf{E}(x, z ; \omega)=\mathbf{e}(x) \exp (i \beta z)$, $\mathbf{H}(x, z ; \omega)=\mathbf{h}(x) \exp (i \beta z)$ и $\mathbf{D}(x, z ; \omega)=\mathbf{d}(x) \exp (i \beta z)$, где $\beta$ - постоянная распространения [26]. Это позволяет свести систему приведенных выше уравнений к двум обыкновенным уравнениям относительно $e_{y}(x)$ и $e_{z}(x)$. Остальные компоненты выражаются через $e_{y}(x)$ и $e_{z}(x)[24-26]$.

Плотность импульса для оптических полей определяется вектором P, усредненным по высокочастотным осцилляциям несущей волны:

$$
\langle\mathbf{P}\rangle=\frac{1}{4 \pi c}\left(\mathbf{e} \times \mathbf{h}^{*}+\mathbf{e}^{*} \times \mathbf{h}\right)
$$

Этот вектор отличается от вектора потока энергии (вектора Пойнтинга) только численным коэффициентом. Используя результаты работы [24], можно найти, что вектор Пойнтинга имеет только одну продольную не равную нулю компоненту, так что $\left\langle P_{x}\right\rangle=\left\langle P_{y}\right\rangle=0$ и $\left\langle P_{z}\right\rangle \neq 0$. Этот известный результат - прямое следствие планарной симметрии рассматриваемой задачи.

В выражении для полного углового момента под интегралом стоит плотность углового момента $\mathbf{j}\left(\mathbf{r}_{0}\right)=\left(\mathbf{r}-\mathbf{r}_{0}\right) \times \mathbf{P}$. После усреднения по высокочастотным колебаниям напряженностей полей усредненная плотность углового момента записывается как $\left\langle\mathbf{j}\left(\mathbf{r}_{0}\right)\right\rangle=\left(\mathbf{r}-\mathbf{r}_{0}\right) \times\langle\mathbf{P}\rangle$. Учитывая, что есть только одна ненулевая проекция усредненной плотности импульса, можно записать

$$
\begin{gathered}
\left\langle j_{x}\left(\mathbf{r}_{0}\right)\right\rangle=\left(y-y_{0}\right)\left\langle P_{z}\right\rangle, \\
\left\langle j_{y}\left(\mathbf{r}_{0}\right)\right\rangle=-\left(x-x_{0}\right)\left\langle P_{z}\right\rangle, \quad\left\langle j_{z}\left(\mathbf{r}_{0}\right)\right\rangle=0 .
\end{gathered}
$$

В силу планарной симметрии задачи можно перенести начало координат в точку $y_{0}$. Величина $\left\langle P_{z}\right\rangle$ является функцией только $x$. Следовательно, $\left\langle j_{x}\left(\mathbf{r}_{0}\right)\right\rangle=y\left\langle P_{z}\right\rangle(x)$ есть нечетная функция относительно переменной $y$ и интегрирование по объему даст нулевое значение проекции на ось $X$ полного углового момента $\left\langle J_{x}\left(\mathbf{r}_{0}\right)\right\rangle=0$. Перенести начало координат в точку $x_{0} \neq 0$ нельзя, поскольку это нарушит планарную симметрии задачи. Таким образом, вектор $\left\langle\mathbf{J}\left(\mathbf{r}_{0}\right)\right\rangle$ лежит в плоскости раздела сред, ортогонален направлению переноса энергии и имеет ненулевую компоненту:

$$
\left\langle J_{y}\left(\mathbf{r}_{0}\right)\right\rangle=-\int x\left\langle P_{z}(x)\right\rangle d x d y d z+x_{0} \int\left\langle P_{z}(x)\right\rangle d x d y d z
$$

Если выбрать область интегрирования в форме параллелепипеда со сторонами $2 L_{x}, 2 L_{y}$ и $2 L_{z}$ так, чтобы начало координат располагалось на поверхности раздела, то справедливо выражение

$$
\left\langle J_{y}\left(\mathbf{r}_{0}\right)\right\rangle=-4 L_{z} L_{y}\left(\int_{-L_{x}}^{L_{x}} x\left\langle P_{z}(x)\right\rangle d x-x_{0} \int_{-L_{x}}^{L_{x}}\left\langle P_{z}(x)\right\rangle d x\right) .
$$

Так как напряженности полей поверхностной волны экспоненциально уменьшаются по мере удаления от границы раздела, можно устремить $L_{x}$ к бесконечности.

Полученный результат не зависит от типа соприкасающихся сред. Важно только, чтобы существовала поверхностная волна, т. е. дисперсионное уравнение должно иметь решение. Например, на границе раздела обычных диэлектриков в линейном приближении поверхностная ТЕ-волна не существует, а ТМ-волна может 
существовать при условии, что диэлектрические проницаемости соприкасающихся сред имеют противоположные знаки. Усредненный момент $\left\langle P_{z}\right\rangle$ определяется следующим выражением: $2 \pi c\left\langle P_{z}\right\rangle=\operatorname{Re}\left(e_{x} h_{y}^{*}\right)-\operatorname{Re}\left(e_{y} h_{x}^{*}\right)$. Для ТМ-волны $e_{y}(x)=0, e_{x}(x) \neq 0$ и $h_{y}(x) \neq 0$. Следовательно, $2 \pi c\left\langle P_{z}\right\rangle=\operatorname{Re}\left(e_{x} h_{y}^{*}\right) \neq 0$ и ТМ-волна обладает угловым моментом.

\section{Вычисление спинового углового момента}

Пусть полупространство при $x<0$ заполнено топологическим изолятором (ТИ), который предполагается изотропным диэлектриком таким, что $D_{y}=\varepsilon_{1} E_{y}$, $D_{z}=\varepsilon_{1} E_{z}$ и $\mu_{1}=1$. Полупространство при $x>0$ заполнено гиперболическим метаматериалом $(Г М)$ - одноосным материалом, у которого главные диэлектрические проницаемости имеют противоположные знаки $[17,18]$. Пусть оптическая ось лежит в плоскости раздела ГМ и ТИ. Компоненты вектора $\mathbf{D}(x, z ; \omega)$ в области, занятой ГМ, определены как $D_{x}=\varepsilon_{o} E_{x}, D_{y}=\varepsilon_{e} E_{y}, D_{z}=\varepsilon_{o} E_{z}$ и $\mu_{2}=1$, где $\varepsilon_{o}$ и $\varepsilon_{e}-$ главные диэлектрические проницаемости.

Система уравнений для $e_{y}(x)$ и $e_{z}(x)$, как и их решения, были найдены в $[24,25]$. Можно использовать полученные там выражения, необходимые для расчета спинового углового момента, опустив подробности. Распределения полей следующие. При $x<0$

$$
\begin{gathered}
e_{x}^{(1)}=(\beta / i q) B e^{q x}, \quad e_{y}^{(1)}=A e^{q x}, \\
e_{z}^{(1)}=B e^{q x}, \quad h_{x}^{(1)}=-\left(\beta / k_{0}\right) A e^{q x}, \\
h_{y}^{(1)}=\left(k_{0} \varepsilon_{1} / i q\right) B e^{q x}, \quad h_{z}^{(1)}=-\left(i q / k_{0}\right) A e^{q x} .
\end{gathered}
$$

При $x>0$

$$
\begin{gathered}
e_{x}^{(2)}=\left(i \beta / p_{2}\right) B_{0} e^{-p_{2} x}, \quad e_{y}^{(2)}=A_{0} e^{-p_{1} x}, \\
e_{z}^{(2)}=B_{0} e^{-p_{2} x}, \quad h_{x}^{(2)}=-\left(\beta / k_{0}\right) A_{0} e^{-p_{1} x}, \\
h_{y}^{(2)}=-\left(i k_{0} \varepsilon_{o} / p_{2}\right) B_{0} e^{-p_{2} x}, \quad h_{z}^{(2)}=\left(i p_{1} / k_{0}\right) A_{0} e^{-p_{1} x} .
\end{gathered}
$$

Дисперсионное уравнение имеет следующий вид:

$$
\left(q+p_{1}\right)\left(\frac{q}{\varepsilon_{1}}+\frac{p_{2}}{\varepsilon_{o}}\right)+\kappa^{2} \frac{p_{2} q}{\varepsilon_{o} \varepsilon_{1}}=0, \quad \kappa=\frac{2 e^{2}}{c \hbar} \Delta \theta,
$$

где параметр $\kappa$ содержит разность топологических чисел $\Delta \theta=\theta^{(1)}-\theta^{(2)}$, которые характеризуют первую и вторую среды. В рассматриваемом случае $\theta^{(1)}=$ $=1+2 n, n-$ целое число, и $\theta^{(2)}=0$. В этих выражениях $q^{2}=\beta^{2}-k_{0}^{2} \varepsilon_{1}>0, p_{1}^{2}=\beta^{2}-k_{0}^{2} \varepsilon_{e}>0, p_{2}^{2}=$ $=\beta^{2}-k_{0}^{2} \varepsilon_{o}>0$ и $p_{1,2}>0$ и $q>0$. Если $\varepsilon_{o}>0$ и $\varepsilon_{1}>0$, уравнение (5) не имеет решений. Следовательно, поверхностной волны не существует. Однако, если $\varepsilon_{o}<0$ и $\varepsilon_{1}>0$, решение уравнения (5) существует в некоторой области частот. Тогда вдоль границы раздела может распространяться волна, у которой поляризация различна по разные стороны границы раздела и изменяется скачком при переходе из одной среды в другую.

Так же как напряженности полей и индукция, векторный потенциал А для рассматриваемой задачи может быть представлен в виде $\mathbf{A}(x, z ; \omega)=\mathbf{a}(x ; \omega) \exp (i \beta z)$, и его амплитуда связана с электрическим полем в выбранной калибровке как $\mathbf{e}(x)=i k_{0} \mathbf{a}(x)$. При $x<0$

$$
\begin{gathered}
a_{x}^{(1)}=-\left(\beta / k_{0} q\right) B e^{q x}, \quad a_{y}^{(1)}=\left(1 / i k_{0}\right) A e^{q x}, \\
a_{z}^{(1)}=\left(1 / i k_{0}\right) B e^{q x},
\end{gathered}
$$

при $x>0$

$$
\begin{gathered}
a_{x}^{(2)}=\left(\beta / k_{0} p_{2}\right) B_{0} e^{-p_{2} x}, \quad a_{y}^{(2)}=\left(1 / i k_{0}\right) A_{0} e^{-p_{1} x}, \\
e_{z}^{(2)}=\left(1 / i k_{0}\right) B_{0} e^{-p_{2} x} .
\end{gathered}
$$

Пусть плотность усредненного по быстрым колебанием во времени спинового момента есть $\mathbf{g}=2 \operatorname{Re}\left(\mathbf{e} \times \mathbf{a}^{*}\right)$, так чтобы $\left\langle\mathbf{S}_{\text {in }}\right\rangle=(4 \pi c)^{-1} \int \mathbf{g} d V$. Используя выражения (3), (4), (6) и (7), можно найти следующие выражения для компонент вектора плотности спинового момента g:

$$
\begin{gathered}
g_{x}^{(1)}=\frac{2 i}{k_{0}}\left(A B^{*}-A^{*} B\right) e^{2 q x}, \\
g_{y}^{(1)}=-\frac{4 \beta}{k_{0} q}|B|^{2} e^{2 q x}, \quad g_{z}^{(1)}=\frac{2 \beta}{k_{0} q}\left(A B^{*}+A^{*} B\right) e^{2 q x}, \\
g_{x}^{(2)}=\frac{2 i}{k_{0}}\left(A B^{*}-A^{*} B\right) e^{-\left(p_{1}+p_{2}\right) x}, \quad g_{y}^{(2)}=\frac{4 \beta}{k_{0} p_{2}}|B|^{2} e^{-2 p_{2} x}, \\
g_{z}^{(2)}=-\frac{2 \beta}{k_{0} p_{2}}\left(A B^{*}+A^{*} B\right) e^{-\left(p_{1}+p_{2}\right) x} .
\end{gathered}
$$

При выводе этих выражений учитывалась непрерывность при $x=0$ касательных компонент напряженности электрического поля. Нормальная компонента электрической индукции и касательные компоненты вектора магнитной индукции при $x=0$ связаны $[19,20,24,25]$ соотношениями

$$
\begin{gathered}
\left(D_{x}^{(1)}-D_{x}^{(2)}\right)=-\kappa B_{x}^{(1)}, \quad\left(H_{y}^{(1)}-H_{y}^{(2)}\right)=\kappa E_{y}^{(1)}, \\
\left(H_{z}^{(1)}-H_{z}^{(2)}\right)=\kappa E_{z}^{(1)} .
\end{gathered}
$$

Используя выражения (3), (4), из (10) можно найти соотношения между $A$ и $B$ :

$$
\left(\frac{\varepsilon_{1}}{q}+\frac{\varepsilon_{0}}{p_{2}}\right) B=\frac{i \kappa}{k_{0}} A, \quad\left(q+p_{1}\right) A=i \kappa k_{0} B .
$$

Из этих уравнений следует дисперсионное уравнение (5). Но отсюда также можно найти связь между величинами $A$ и $B$, которые являются касательными компонентами напряженности электрического поля при $x=0$. Результат запишется как

$$
B=\frac{i \kappa q p_{2}}{k_{0}\left(p_{2} \varepsilon_{1}+q \varepsilon_{o}\right)} A \equiv i \rho A .
$$


Надо вспомнить, что для существования поверхностной волны необходимо, чтобы $\varepsilon_{o}<0$ и $\varepsilon_{2}>0$. Из (5) следует, что $\left(q+p_{1}\right)\left(q\left|\varepsilon_{o}\right|-p_{2} \varepsilon_{1}\right)=\kappa^{2} p_{2} q$. Следовательно, знаменатель в (12) не обращается в нуль.

Учитывая (11), можно получить выражения для компонент плотности спинового момента $\mathbf{g}$ :

$$
\begin{gathered}
g_{x}^{(1)}=\frac{4 \rho}{k_{0}}|A|^{2} e^{2 q x}, \\
g_{y}^{(1)}=-\frac{4 \beta \rho^{2}}{k_{0} q}|A|^{2} e^{2 q x}, \quad g_{z}^{(1)}=0, \\
g_{x}^{(2)}=\frac{4 \rho}{k_{0}}|A|^{2} e^{-\left(p_{1}+p_{2}\right) x}, \\
g_{y}^{(2)}=\frac{4 \beta \rho^{2}}{k_{0} p_{2}}|A|^{2} e^{-2 p_{2}} x, \quad g_{z}^{(2)}=0 .
\end{gathered}
$$

В отличие от случая поверхностных плазмонполяритонов [8,9] здесь имеется ненулевая нормальная проекция вектора спинового момента. Касательная проекция вектора спинового момента знакопеременная. В точке $x=0$ векторы $\mathbf{g}^{(1)}$ и $\mathbf{g}^{(2)}$ неколлинеарные. Если определить углы $\gamma_{1}$ и $\gamma_{2}$ соотношениями

$$
\tan \gamma_{j}=\frac{\left|g_{y}^{(j)}(0)\right|}{g_{x}^{(j)}(0)}, \quad j=1,2,
$$

то можно найти, что $\tan \gamma_{1}=(\beta / q) \rho, \tan \gamma_{2}=\left(\beta / p_{2}\right) \rho$. Угол $\gamma$ между векторами $\mathbf{g}^{(1)}$ и $\mathbf{g}^{(2)}$ определяется по формуле

$$
\tan \gamma=\tan \left(\gamma_{1}+\gamma_{2}\right)=\frac{\tan \gamma_{1}+\tan \gamma_{2}}{1-\tan \gamma_{1} \tan \gamma_{2}} .
$$

Отсюда

$$
\tan \gamma=\frac{\beta\left(q+p_{2}\right) \rho}{q p_{2}-\beta^{2} \rho^{2}} .
$$

Из этого выражения видно, то угол $\gamma$ не превышает $\pi / 2$.

Если среды топологически неразличимы, то $\Delta \theta=$ $=\theta^{(1)}-\theta^{(2)}=0$, и уравнения (11) приводят к двум соотношениям: $\left(q+p_{1}\right) A=0$ (откуда следует, что $A=0)$ и дисперсионному уравнению для поверхностной ТМ-волны

$$
\left(\frac{\varepsilon_{1}}{q}-\frac{\left|\varepsilon_{o}\right|}{p_{2}}\right)=0 .
$$

Величина $B$ имеет смысл напряженности поля $e_{z}^{(1)}(0)=$ $=e_{z}^{(2)}(0)$. Из приведенных выше выражений для плотностей спинового момента (8), (9) следует, что

$$
\begin{array}{r}
g_{x}^{(1)}=0, \quad g_{y}^{(1)}=-\frac{4 \beta}{k_{0} q}|B|^{2} e^{2 q x}, \quad g_{z}^{(1)}=0, \\
g_{x}^{(2)}=0, \quad g_{y}^{(2)}=\frac{4 \beta}{k_{0} p_{2}}|B|^{2} e^{-2 p_{2} x}, \quad g_{z}^{(2)}=0 .
\end{array}
$$

Таким образом, вектор спинового момента лежит в плоскости раздела обычных сред. Интересно, что получить этот результат прямым предельным переходом $\kappa \rightarrow 0$ в (13) и (14) не удастся. Тут можно предположить аналогию с фазовым переходом первого рода, где $\kappa$ играет роль температуры, а $g_{x}^{(1)}(0)$ - параметр порядка. Важно отметить, что величина $g_{x}^{(1)}(0)=g_{x}^{(0)}(0)$ пропорциональна топологическому числу $\theta^{(1)}=1+2 n$, где $n$ - целое число. Величины $g_{y}^{(1)}(0)$ и $g_{z}^{(1)}(0)$, как следует из (13) и (14), пропорциональны $\left(\theta^{(1)}\right)^{2}$. Это означает квантование касательной компоненты спинового углового момента. Когда вместо ТИ взята обычная среда, то компоненты спинового углового момента (15) однозначны - квантования нет. Следовательно, ТИ навязывает топологическое квантование максимальному значению напряженности электрического поля на границе раздела.

\section{Заключение}

В настоящей работе была рассмотрена поверхностная волна и вычислен ее спиновый угловой момент, который является внутренней характеристикой электромагнитного поля волны. В качестве конкретного примера рассматривалась граница раздела между ТИ и ГМ. Основным результатом является то обстоятельство, что спиновый угловой момент не лежит в плоскости границы раздела сред. Появление нормальной компоненты спинового углового момента обусловлено свойствами ТИ. На его поверхности возникает поверхностный ток и наводится заряд, которых нет в обычных диэлектриках. Это приводит к изменению условий непрерывности для нормальных компонент электрической индукции и касательных компонент магнитной индукции. В результате меняется поляризация волны при переходе через границу раздела. Отдельно ТЕ- и ТМ-волн нет, но имеет место гибридная волна, у которой все компоненты поля ненулевые. Формально это приводит к появлению ненулевой нормальной компоненты спинового углового момента. Надо заметить, что присутствие ТИ проявляется в дискретности этой компоненты:

$$
\begin{gathered}
g_{x}^{(1)}(0)=g_{x}^{(2)}=\frac{2 e^{2}}{c \hbar} \frac{4 q p_{2}}{k_{0}^{2}\left(p_{2} \varepsilon_{1}+q \varepsilon_{o}\right)}|A|^{2}(1+2 n), \\
n=0,1,2, \ldots
\end{gathered}
$$

Поскольку спиновый угловой момент есть часть орбитального момента, полученное топологическое квантование будет проявляться в полнм угловом моменте. Если использовать (2)-(4) и вычислить входящий в (2) интеграл при $L_{x} \rightarrow \infty$, то можно убедиться, что полный угловой момент содержит слагаемое, пропорциональное $(1+2 n)^{2}$.

Хотя в качестве одной из сред рассматривался ГМ, возникновение нормальной компоненты спинового углового момента может происходить, если ГМ заменить металлом. Тогда поверхностный плазмон-поляритон будет переносить спиновый момент, имеющий нормальную компоненту. 
Рассмотрен случай оптически линейных сред. В [24] было показано, что поверхностная волна на границе кубически нелинейного диэлектрика и ТИ может существовать, если переносимая ею мощность превысит некоторый порог. Можно ожидать, что спиновый угловой момент и в этом случае будет иметь нормальную компоненту. Как величина этой компоненты будет зависеть от мощности поверхностной волны - предстоит изучить.

Выбор кулоновской калибровки при вычислении спинового углового момента сделало эти вычисления калибровочно-неинвариантными. Это стандартная проблема выделения спинового углового момента из полного углового момента.

Исследование выполнено при поддержке Российского научного фонда (проект № 18-02-00921).

\section{Список литературы}

[1] O'Neil A.T., MacVicar I., Allen L., Padgett M.J. // Phys. Rev. Lett. 2002. V. 88. P. 061101.

[2] Barnett S.M. // J. Mod. Opt. 2010. V. 57. N 14-15. P. 1339.

[3] Bialynicki-Birula I., Bialynicka-Birula Z. // J. Opt. 2011. V. 13. P. 064014.

[4] Barnett S.M.,. Allen L, Cameron R.P., Gilson C.R., Padgett M.J., Speirits F.C., Yao A.M. // J. Opt. 2016. V. 18. P. 064004.

[5] Батыгин В.В., Топтыгин И.Н. Сборник задач по электродинамике и специальной теории относительности. СПб.: Лань, 2010. 256 с.

[6] Aiello A., Marquardt Ch., Leuchs G. // Phys. Rev. A. 2010. V. 81. P. 053838.

[7] Bliokh K.Y., Bekshaev A.Y., Nori F. Электронный pecypc. Режим доступа: arXiv:1308.0547 [physics. optics]

[8] Bliokh K.Y., Nori F. // Phys. Rev. A. 2012. V. 85. P. 061801(R).

[9] Bliokh K.Y., Nori F. // Phys. Rep. 2015. V. 592. P. 1 ?38.

[10] Bliokh K.Y., Bekshaev A.Y., Nori F. // New J. Phys. 2017. V. 19. P. 063021.

[11] Berini P. // Adv. Opt. Photon. 2009. V. 1. N 3. P. 484.

[12] Wang Y., Plummer E.W., Kempa K. // Adv. Phys. 2011. V. 60. N 5. P. 799.

[13] Silveirinha M., Engheta N. // Phys. Rev. B. 2007. V. 75. P. 075119.

[14] Alekseyev L.V., Narimanov E.E., Tumkur T., Li H., Barnakov Yu.A., Noginov M.A. // Appl. Phys. Lett. 2010. V. 97. P. 131107.

[15] Konstantinidis K., Feresidis A.P. // J. Opt. 2015. V. 17. P. 105104.

[16] Starko-Bowes R., Atkinson J., Newman W., Hu H., Kallos T., Palikaras G., Fedosejevs R., Pramanik S., Jacob Z. // JOSA. B. 2015. V. 32. N 10. P. 2074.

[17] Elser J., Wangberg R., Podolskiy V.A., Narimanov E.E. // Appl. Phys. Lett. 2006. V. 89. P. 261102.

[18] Drachev V.P., Podolskiy V.A., Kildishev A.V. // Opt. Express. 2013. V. 21. N 12. P. 15048.

[19] Hasan M.Z., Kane C.L. // Rev. Mod. Phys. 2010. V. 82. P. 3045.

[20] Xiao-Liang Qi, Shou-Cheng Zhang // Rev. Mod. Phys. 2011. V. 83. P. 1057.
[21] Wang-Kong Tse, MacDonald A.H. // Phys. Rev. Lett. 2010. V. 105. P. 057401.

[22] Karch A. // Phys. Rev. B. 2011. V. 83. P. 245432.

[23] Huerta L. // Phys. Rev. D. 2016. V. 94. P. 125021.

[24] Ляшко Е.И., Маймистов А.И. // Опт. и спектр. 2016. T. 121. № 4. C. 671.

[25] Lyashko E.I., Maimistov A.I., Gabitov I.R. Электронный реcypc. Режим доступа: arXiv: 1706.05951v1 [physics. optics].

[26] Маймистов А.И., Ляшко Е.И. // Известия РАН. Сер.физ. 2018. T. 82. № 1. С. 27.

[27] Integrated Optics / Ed. by Tamir T. Berlin: Springer, 1983. 